\title{
A novel training simulator for portable ultrasound identification of incorrect newborn endotracheal tube placement - observational diagnostic accuracy study protocol
}

Hasan S. Merali' ${ }^{1}$, Mark O. Tessaro ${ }^{2}$, Khushboo Q. Ali ${ }^{3}$, Shaun K. Morris ${ }^{4}$, Sajid B. Soofi ${ }^{3}$ and Shabina Ariff ${ }^{3 *}$

\begin{abstract}
Background: Endotracheal tube (ETT) placement is a critical procedure for newborns that are unable to breathe. Inadvertent esophageal intubation can lead to oxygen deprivation and consequent permanent neurological impairment. Current standard-of-care methods to confirm ETT placement in neonates (auscultation, colorimetric capnography, and chest $\mathrm{x}$-ray) are time consuming or unreliable, especially in the stressful resuscitation environment. Point-of-care ultrasound (POCUS) of the neck has recently emerged as a powerful tool for detecting esophageal ETTs. It is accurate and fast, and is also easy to learn and perform, especially on children.

Methods: This will be an observational diagnostic accuracy study consisting of two phases and conducted at the Aga Khan University Hospital in Karachi, Pakistan. In phase 1, neonatal health care providers that currently perform standardof-care methods for ETT localization, regardless of experience in portable ultrasound, will undergo a two-hour training session. During this session, providers will learn to detect tracheal vs. esophageal ETTs using POCUS. The session will consist of a didactic component, hands-on training with a novel intubation ultrasound simulator, and practice with stable, ventilated newborns. At the end of the session, the providers will undergo an objective structured assessment of technical skills, as well as an evaluation of their ability to differentiate between tracheal and esophageal endotracheal tubes. In phase 2, newborns requiring intubation will be assessed for ETT location via POCUS, at the same time as standard-of-care methods. The initial 2 months of phase 2 will include a quality assurance component to ensure the POCUS accuracy of trained providers. The primary outcome of the study is to determine the accuracy of neck POCUS for ETT location when performed by neonatal providers with focused POCUS training, and the secondary outcome is to determine whether neck POCUS is faster than standard-of-care methods.
\end{abstract}

Discussion: This study represents the first large investigation of the benefits of POCUS for ETT confirmation in the sickest newborns undergoing intubations for respiratory support.

Trial registration: ClinicalTrials.gov Identifier: NCT03533218. Registered May 2018.

Keywords: Newborn resuscitation, Point of care ultrasound (POCUS), Simulation, Pakistan, Endotracheal tube (ETT), Intubation

\footnotetext{
* Correspondence: shabina.ariff@aku.edu

${ }^{3}$ Department of Paediatrics \& Child Health, Aga Khan University, Stadium

Road, Karachi 74800, Pakistan

Full list of author information is available at the end of the article
}

(c) The Author(s). 2019 Open Access This article is distributed under the terms of the Creative Commons Attribution 4.0 International License (http://creativecommons.org/licenses/by/4.0/), which permits unrestricted use, distribution, and reproduction in any medium, provided you give appropriate credit to the original author(s) and the source, provide a link to the Creative Commons license, and indicate if changes were made. The Creative Commons Public Domain Dedication waiver (http://creativecommons.org/publicdomain/zero/1.0/) applies to the data made available in this article, unless otherwise stated. 


\section{Background}

Two-thirds of global neonatal deaths occur in just 10 countries and the majority occur in Asia [1]. Pakistan has the highest neonatal mortality of Asian nations with 46.6 deaths per 1000 live births [2].

Early neonatal deaths account for $75 \%$ of all neonatal deaths, and preventing these deaths depends on attention to the causes of death that are unique to the first week of life, particularly birth asphyxia [3], which accounts for $23 \%$ of global neonatal deaths [1]. If not fatal, birth asphyxia can cause severe neurologic impairment including motor and cognitive disabilities [4].

During the first seconds after birth, radical and rapid cardiopulmonary adaptation to the extra-uterine environment must occur for neonates to survive $[5,6]$. The most important physiologic change is establishment of independent respiration [7]. It is during this period that approximately $10 \%$ neonates require some level of resuscitation [8], and the more premature the newborn, the higher the likelihood that intubation is required [9]. Tracheal intubation is performed frequently in delivery rooms and Neonatal Intensive Care Units (NICU). Neonatal intubation is a critical and time-sensitive procedure, as failure deprives the highest risk newborns of oxygen [10]. Prompt recognition of inadvertent esophageal intubation is vital to prevent the mortality or permanent neurologic consequences of neonatal hypoxia $[11,12]$. Unfortunately, neonatal intubation is challenging due to the unique airway anatomy of newborns, and inadvertent esophageal placement of the ETT is common. In a recent multi-center study examining intubations both in the NICU and delivery room, the authors found that esophageal intubation occurred between 1 and $12 \%$ of the time [13]. It occurs with even greater frequency with junior trainees often with delayed recognition and correction [11].

Current methods to detect esophageal intubation are imperfect. Chest auscultation is notoriously unreliable [14], especially during noisy and chaotic neonatal resuscitations, where one study found it requires an average of $77 \mathrm{~s}$ [15]. Capnography improves upon clinical exam alone, but requires vigorous tissue oxygenation and cardiopulmonary function, [15] which is not present for most peri-arrest neonates. Capnography is usually performed with a qualitative colorimetric device, which exhibits a sensitivity of only $65 \%$ for correct airway placement, thus predisposing many correctly intubated neonates to unnecessary re-intubation attempts during which they experience no oxygen delivery and potential airway trauma [16, 17]. These devices also generate false-positive results if they contact secretions or epinephrine, substances commonly suctioned or delivered through ETTs [18]. Capnographic accuracy is improved with quantitative continuous wave-form monitors, but these are expensive, take up to $30 \mathrm{~s}$ to generate a result, and still produce false-negative results in peri-arrest patients.

POCUS of the anterior neck is increasingly used by emergency physicians and anesthesiologists to detect esophageal intubation. It is more accurate and faster than physical examination and capnography, with adult meta-analyses reporting sensitivities of 93-98\%, specificities of $97-98 \%$, and an average performance time of $9 \mathrm{~s}$ $[19,20]$. The technique is easy to learn, with trainees serving as the ultrasound operators in most studies [20]. This technique may be most easily applied in children, with reported sensitivities and specificities of $100 \%$, even in children undergoing chest compressions [21].

POCUS presents significant advantages over physical examination and capnography during resuscitation. Its direct cross-sectional imaging of the neck is not affected by cardiopulmonary function, chest compressions, or environmental noise [22]. Due to its quick result, it also prevents the gastric distention and vomiting that can occur from ventilation through an esophageal ETT [23].

Despite its prevalence in adult and pediatric resuscitations, intubation POCUS has not yet translated to neonatology. Only two studies with small sample sizes exist on the detection of esophageal intubation in neonates using portable ultrasound. A recent Cochrane review on methods to determine ETT position in neonates identified portable ultrasound as a promising modality in need of further study [24]. Neonatology services increasingly utilize POCUS for other assessment and diagnostic applications, making uptake of intubation POCUS feasible in neonatal resuscitations.

This study will determine whether neonatal providers that undergo a two-hour training session with a novel intubation POCUS simulator can then accurately detect tracheal vs. esophageal ETTs using POCUS, and generate a more rapid result than standard-of-care methods. This novel intubation POCUS simulator is inexpensive and can be made from simple materials found commonly throughout the world. This study will provide training to the neonatal providers currently performing standard-of-care methods for ETT localization, regardless of POCUS experience, including attending physicians (staff specialists), junior and senior medical trainees, and senior nurses. This study represents the first step in translating the established benefits of intubation POCUS to the sickest newborns undergoing intubation for respiratory support.

\section{Methods/design}

\section{Study overview and population}

This will be an observational diagnostic accuracy study with a training phase (phase 1) and an assessment phase (phase 2). In phase 1, we will train neonatal providers to detect esophageal versus tracheal ETTs using the intubation POCUS simulator, and then assess their accuracy 
using evaluation tools. In phase 2, newborns requiring intubation in the NICU or labour room/operating room (LR/OR) at The Aga Khan University Hospital, Karachi (AKUH), will have ETT location assessed by POCUS at the same time as current hospital standard-of-care methods (auscultation, colorimetric capnography, and chest $\mathrm{x}$-ray. The total duration of the study will be 18 months.

The Aga Khan University Hospital in Karachi, Pakistan, is an academic tertiary care hospital with a level III NICU. Each year over 5000 deliveries take place at AKUH and approximately $12 \%$ of those newborns are admitted to the NICU, of which approximately $70 \%$ will require intubation. In addition, approximately $50 \%$ of the admitted newborns are transferred from regional hospitals.

\section{Recruitment and eligibility criteria}

Participants for phase 1 of the study will include healthcare providers who currently perform standard-of-care ETT location assessment in the delivery room or NICU at AKUH. This includes neonatologists, neonatology fellows, pediatric residents and senior nurses. These individuals will be identified by the NICU clinical director. Participation is voluntary and may be withdrawn at any time. A detailed informed consent document will be reviewed with each participant prior to the training session and subsequent testing phase.

For phase 2, any newborn needing intubation in the delivery room or NICU will be eligible for the study.
Newborns will be excluded if they have abnormal anatomy of the oropharynx or airway, or if the family declines to consent. Importantly, newborns who require emergency intubation at birth will be receive POCUS during resuscitation, as it will not be possible to obtain prior consent. The family will be approached at a later time when they would be able to offer a deferred informed consent, and will decide to have their newborn's data either included or withdrawn from the study. Neonates requiring non-emergency intubation will be enrolled only if the family provides prior informed consent.

\section{Ultrasound simulator}

In this study, we will utilize a novel, low-cost airway ultrasound simulator which is made on a stove by mixing beef gelatin powder, orange colored psyllium fiber, and water [25]. It costs approximately $\$ 2$ USD for the materials required to create the simulator. It then requires overnight refrigeration and a simple procedure using a $5 \mathrm{ml}$ syringe to create a simulated trachea and esophagus (see Fig. 1).

\section{Ultrasound equipment}

In this study, we will utilize the Philips Lumify USB ultrasound system chosen for its compact design, image quality and portability. Specifically, the L12-4 linear array transducer will be utilized. The depth of the probe will be set at $2.5 \mathrm{~cm}$. The probe will be connected to an
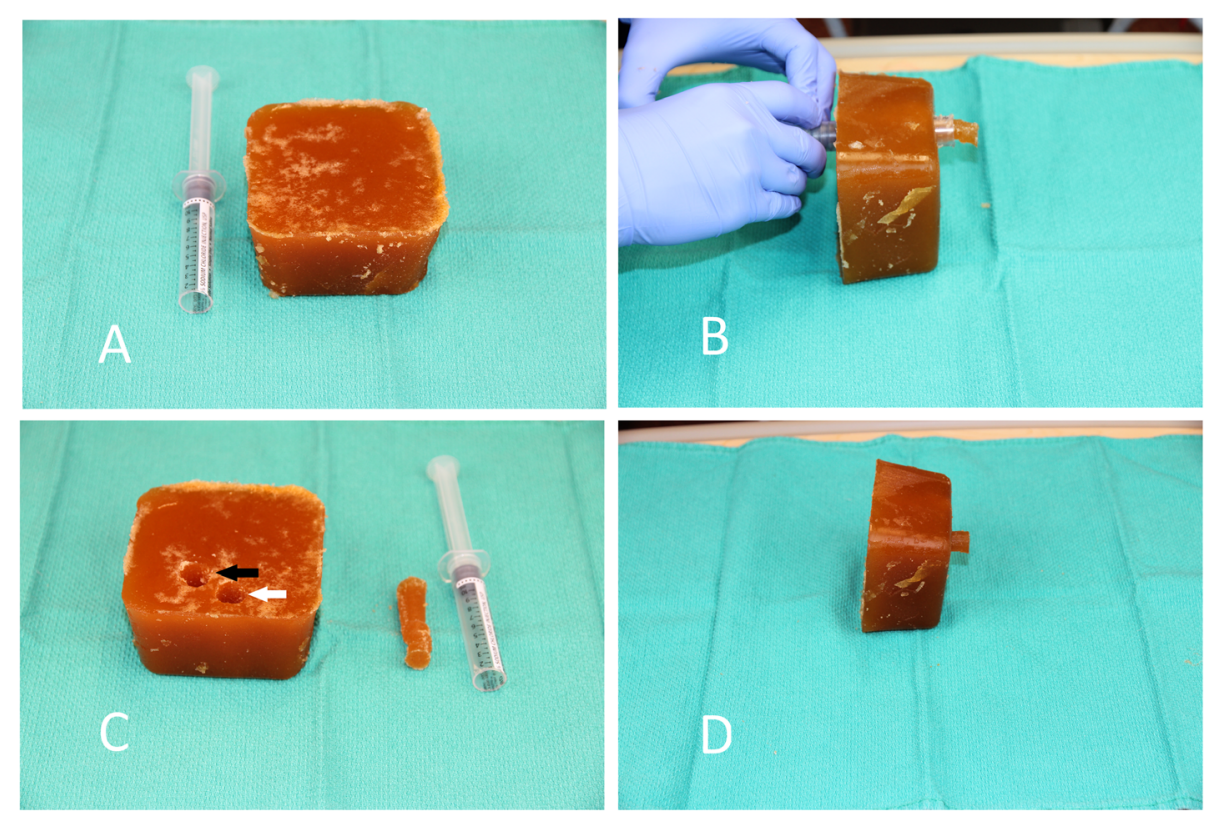

Fig. 1 Low-cost ultrasound simulator. a The beef gelatine and psyllium fiber block, with cut-off $10 \mathrm{~mL}$ syringe barrel. b With cut-off $10 \mathrm{~mL}$ syringe barrel being used to create staggered hollow cylinders within the block. c Block with staggered hollow cylinders, with retained plug from cylinder creation. More superior cylinder is simulated trachea (white arrow), and more inferior cylinder is simulated esophagus (black arrow). d Plug partial insertion into more inferior cylinder (model esophagus) 
Android tablet, version 4.5 this system allows for videos between $1 \mathrm{~s}$ and $10 \mathrm{~min}$ to be captured.

\section{Intervention - phase 1}

The first phase is training of the healthcare providers to sonographically identify correct tracheal and incorrect esophageal placement of an ETT. This two-hour session will be conducted by a study co-investigator with paediatric airway POCUS expertise. It will begin with a

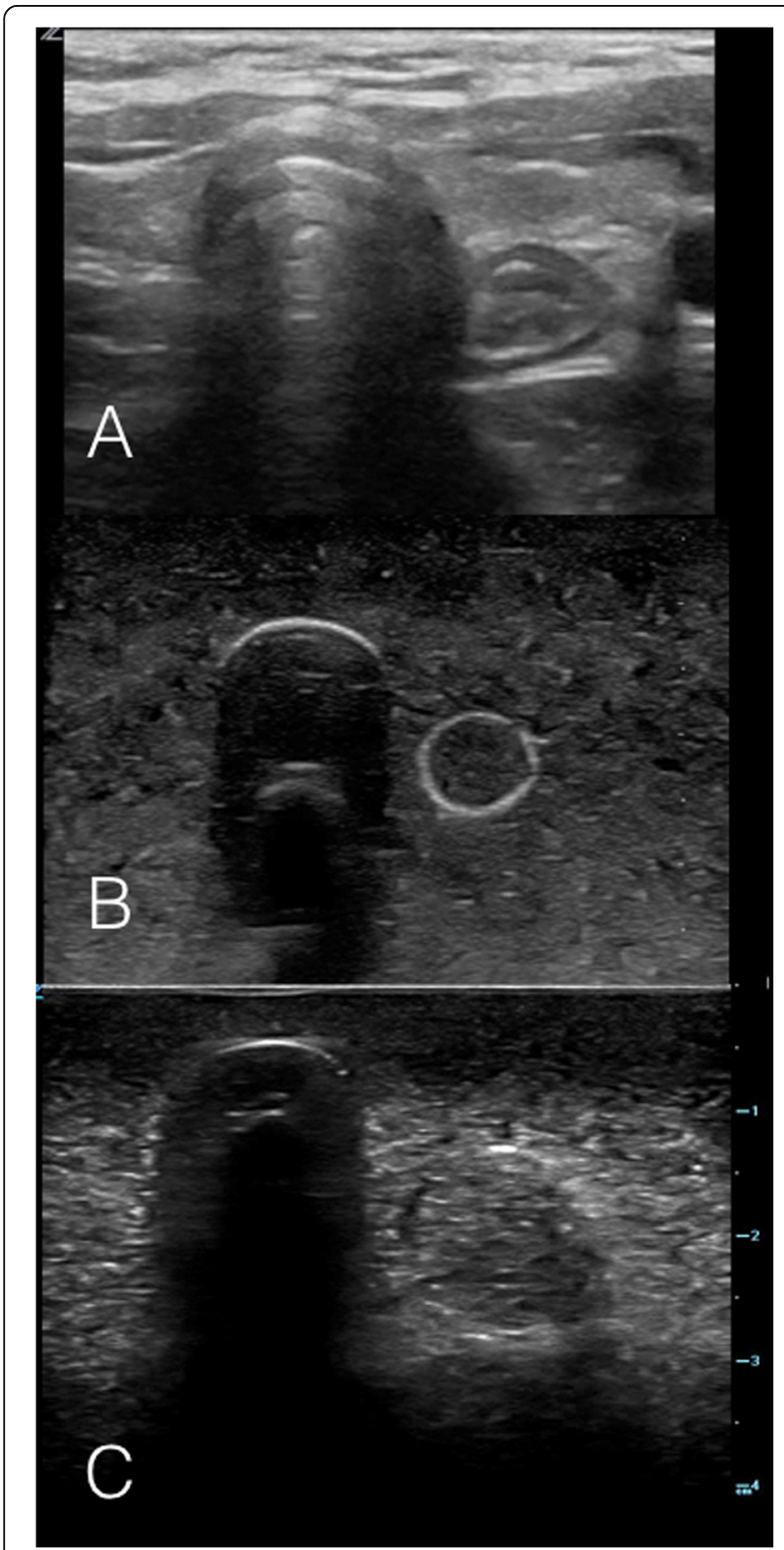

Fig. 2 Ultrasound images on the model: Tracheal Intubation. a Static ultrasound image of a patient with the ETT in the trachea, with linear ultrasound probe held in transverse orientation over the anterior neck at the level of the sternal notch. b \& c Static ultrasound images of beef gelatine model with plug inserted into simulated esophagus, simulating the ultrasound appearance of a tracheal intubation didactic presentation, followed by demonstration using a newborn doll (to demonstrate gel use and probe positioning) and the airway ultrasound simulator (to demonstrate POCUS image acquisition and interpretation (see Figs. 2 and 3). After a period of practicing gel use and probe positioning with the newborn doll, the healthcare providers will then demonstrate these same skills on stable intubated and non-intubated patients in the NICU. The instructor will assess these skills and then provide feedback to the healthcare providers. The healthcare providers will then practice ETT location detection using the airway ultrasound simulators (see Fig. 4). Finally, the healthcare providers will undergo an objective structured assessment of technical skills (see Fig. 5), as well as an evaluation of their ability to differentiate between tracheal and esophageal ETTs (see Fig. 6).

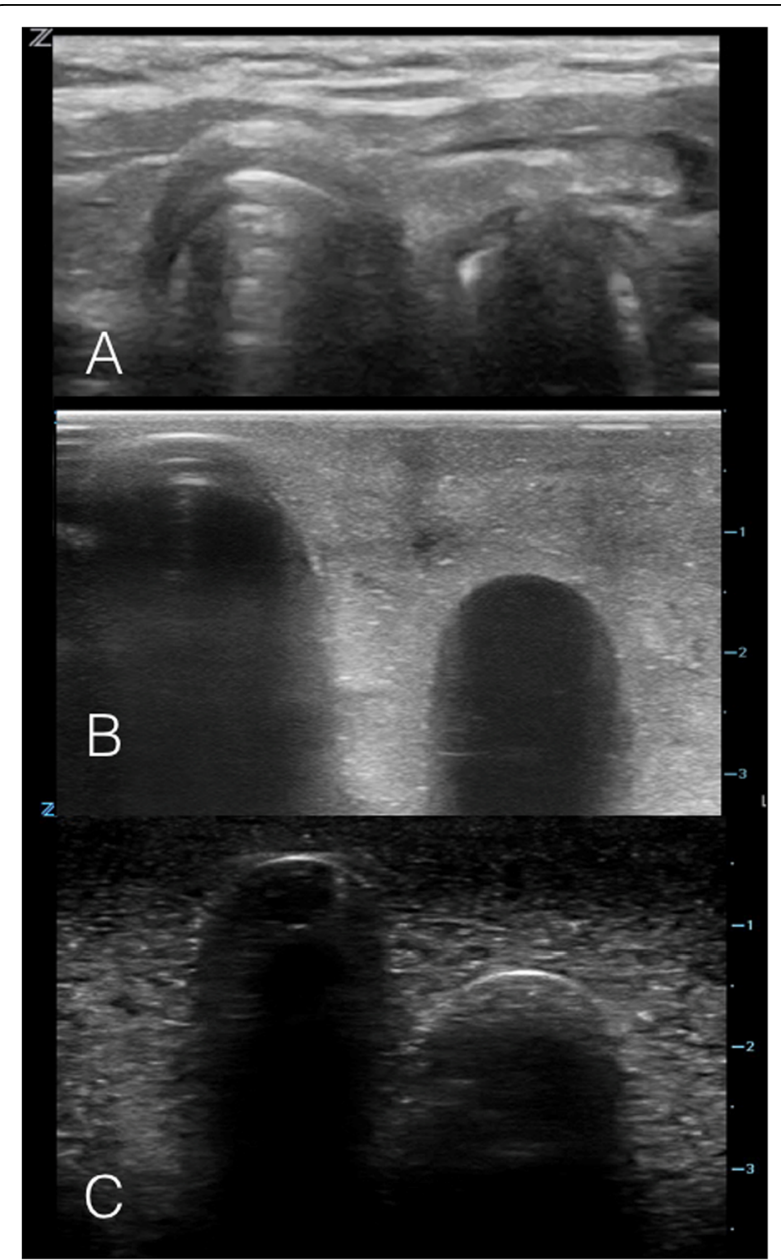

Fig. 3 Ultrasound images on the model: Esophageal Intubation. a Static ultrasound image of an esophageal intubation in a patient, with linear ultrasound probe held in transverse orientation over the anterior neck at the level of the sternal notch. b \& c Static ultrasound images of beef gelatine model with plug removed from simulated esophagus, simulating the ultrasound appearance of an esophageal intubation 


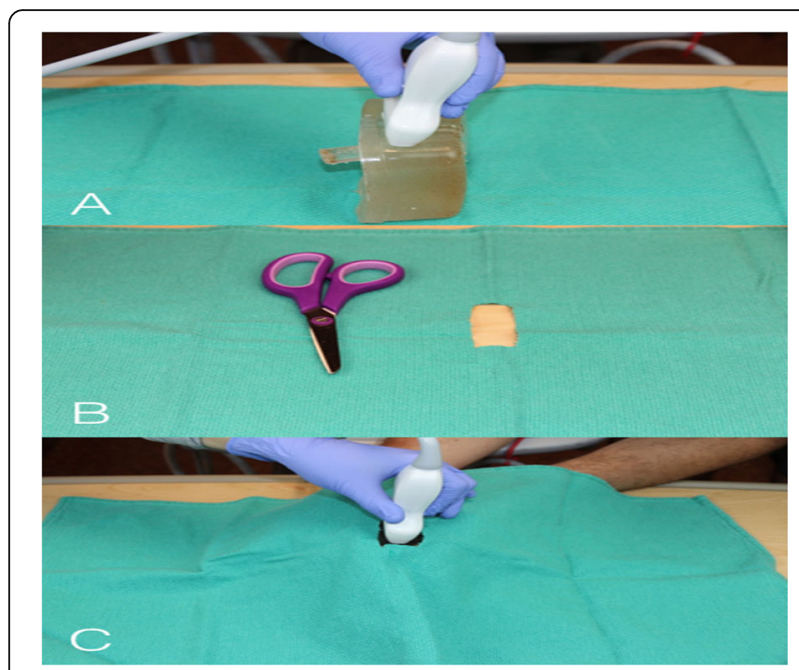

Fig. 4 Placement of the probe on the Simulator. a The first image shows placement of linear probe over the side of the model to obtain images in transverse plane. b Demonstrates a towel with a square hole of $1 \times 3 \mathrm{~cm}$ and $\mathbf{c}$ Shows the towel is placed over the beef gelatin model to hide the location of the plug from the ultrasound operator
Only those participants who pass both these examinations will move on to phase 2 . Providers who do not obtain passing scores will be allowed to repeat the training course one additional time prior to re-examination.

\section{Assessment - phase 2}

The second phase of this project is an observational diagnostic accuracy study. Newborns at AKUH receiving intubation will be assessed by POCUS at the same time as standard-of-care methods for ETT location (auscultation, colorimetric capnography, and chest $x$-ray), and the accuracy of the healthcare providers using POCUS will be determined. The time required to assess ETT location will also be compared between POCUS and standard-ofcare methods.

There will be 2 POCUS machines available, one in the NICU and the other in the LR/OR. At the time of delivery, a clinical team consisting of a neonatology fellow, a pediatric resident, and a staff nurse are available to receive the newborn. If a newborn requires intubation, this will be performed by the fellow or resident, who will assess tracheal versus esophageal ETT location via

\begin{tabular}{|c|c|}
\hline $\begin{array}{l}\text { Preparation for procedure } \\
\text { Hand Washing/Gloves/organize equipment }\end{array}$ & $\begin{array}{l}0 . \ldots \ldots \ldots . . . . \text { Not done OR done incorrectly } \\
1 \ldots \ldots \ldots . . . . \text { Done correctly }\end{array}$ \\
\hline Transducer cleaning prior to scanning & $\begin{array}{l}0 \ldots \ldots \ldots . . . \text { Not done OR done incorrectly } \\
1 \ldots \ldots \ldots . . . . \text { Done correctly }\end{array}$ \\
\hline Turning on the POCUS device & $\begin{array}{l}0 \ldots \ldots . . . . . . \text { Not done OR done incorrectly } \\
\text { 1........... Done correctly }\end{array}$ \\
\hline Entering subject (mannikin) identifier in POCUS device & $\begin{array}{l}0 \ldots \ldots \ldots . . . \text { Not done OR done incorrectly } \\
1 \ldots \ldots \ldots . . . . \text { Done correctly }\end{array}$ \\
\hline $\begin{array}{l}\text { Placement of the POCUS device relative to the mannikin } \\
\text { to allow easy visualization of both }\end{array}$ & $\begin{array}{l}0 \ldots \ldots \ldots . . . . \text { Not done OR done incorrectly } \\
1 \ldots \ldots \ldots . . . . \text { Done correctly }\end{array}$ \\
\hline $\begin{array}{l}\text { Demonstrated POCUS scan covers appropriate areas of } \\
\text { mannikin's neck }\end{array}$ & $\begin{array}{l}0 \ldots \ldots \ldots . . . . \text { Not done OR done incorrectly } \\
1 \ldots \ldots \ldots . . . . \text { Done correctly }\end{array}$ \\
\hline Appropriate volume and distribution of gel on simulator & $\begin{array}{l}0 \ldots \ldots \ldots . . . . \text { Not done OR done incorrectly } \\
1 \text { 1........... Done correctly }\end{array}$ \\
\hline Appropriate gain settings & $\begin{array}{l}0 \ldots \ldots \ldots . . . \text { Not done OR done incorrectly } \\
1 \ldots \ldots \ldots . . . . \text { Done correctly }\end{array}$ \\
\hline Appropriate depth settings & $\begin{array}{l}0 \ldots \ldots \ldots . . . . \text { Not done OR done incorrectly } \\
1 \ldots \ldots \ldots . . . . \text { Done correctly }\end{array}$ \\
\hline Appropriate transducer orientation & $\begin{array}{l}0 \ldots \ldots \ldots . . . . \text { Not done OR done incorrectly } \\
1 \ldots \ldots \ldots . . . . \text { Done correctly }\end{array}$ \\
\hline Images appropriately recorded & $\begin{array}{l}0 \ldots \ldots \ldots . . . . \text { Not done OR done incorrectly } \\
1 \ldots \ldots \ldots . . . . \text { Done correctly }\end{array}$ \\
\hline Turning off the POCUS device & $\begin{array}{l}0 \ldots \ldots \ldots . . . . \text { Not done OR done incorrectly } \\
\text { 1........... Done correctly }\end{array}$ \\
\hline Transducer cleaning after scanning & $\begin{array}{l}0 \ldots \ldots \ldots . . . \text { Not done OR done incorrectly } \\
1 \ldots \ldots \ldots . . . . \text { Done correctly }\end{array}$ \\
\hline Overall performance & 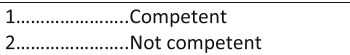 \\
\hline
\end{tabular}

Fig. 5 Objective Structured Assessment of Technical Skills 


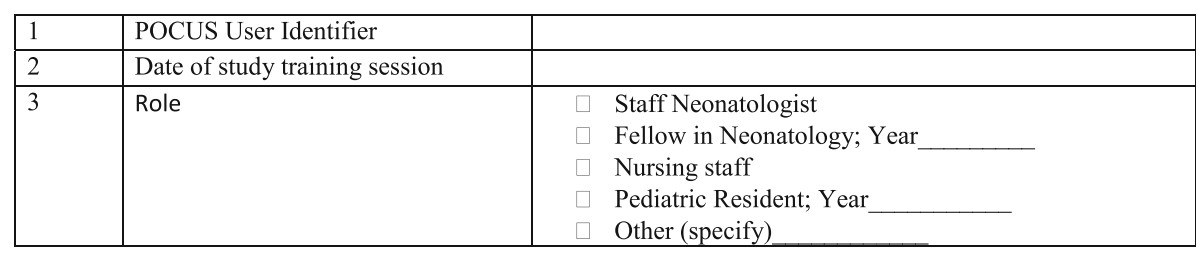

\begin{tabular}{|c|c|c|c|c|c|c|c|}
\hline Test \# & \begin{tabular}{|l|} 
Simulator \\
State
\end{tabular} & $\begin{array}{l}\text { Time at } \\
\text { Start }\end{array}$ & $\begin{array}{l}\text { Air } \\
\text { columns }\end{array}$ & $\begin{array}{l}\text { Esophagus } \\
\text { appearance }\end{array}$ & $\begin{array}{l}\text { Interp } \\
\text { ETT p }\end{array}$ & $\begin{array}{l}\text { ion of } \\
\text { on }\end{array}$ & $\begin{array}{l}\text { Time at POCUS } \\
\text { Interpretation }\end{array}$ \\
\hline 1 & $\begin{array}{l}\text { Trach } \\
\text { Esoph }\end{array}$ & & $\begin{array}{l}1 \\
2\end{array}$ & $\begin{array}{c}\text { Empty } \\
\text { ETT Seen }\end{array}$ & Trach & Esoph & \\
\hline 2 & $\begin{array}{l}\text { Trach } \\
\text { Esoph }\end{array}$ & & $\begin{array}{l}1 \\
2\end{array}$ & $\begin{array}{c}\text { Empty } \\
\text { ETT Seen }\end{array}$ & Trach & Esoph & \\
\hline 3 & $\begin{array}{l}\text { Trach } \\
\text { Esoph }\end{array}$ & & $\begin{array}{l}1 \\
2\end{array}$ & $\begin{array}{c}\text { Empty } \\
\text { ETT Seen }\end{array}$ & Trach & Esoph & \\
\hline 4 & $\begin{array}{l}\text { Trach } \\
\text { Esoph }\end{array}$ & & $\begin{array}{l}1 \\
2\end{array}$ & $\begin{array}{c}\text { Empty } \\
\text { ETT Seen }\end{array}$ & Trach & Esoph & \\
\hline 5 & $\begin{array}{l}\text { Trach } \\
\text { Esoph }\end{array}$ & & $\begin{array}{l}1 \\
2\end{array}$ & $\begin{array}{c}\text { Empty } \\
\text { ETT Seen }\end{array}$ & Trach & Esoph & \\
\hline 6 & $\begin{array}{l}\text { Trach } \\
\text { Esoph }\end{array}$ & & $\begin{array}{l}1 \\
2\end{array}$ & $\begin{array}{c}\text { Empty } \\
\text { ETT Seen }\end{array}$ & Trach & Esoph & \\
\hline 7 & $\begin{array}{l}\text { Trach } \\
\text { Esoph }\end{array}$ & & $\begin{array}{l}1 \\
2\end{array}$ & $\begin{array}{c}\text { Empty } \\
\text { ETT Seen }\end{array}$ & Trach & Esoph & \\
\hline 8 & $\begin{array}{l}\text { Trach } \\
\text { Esoph }\end{array}$ & & $\begin{array}{l}1 \\
2\end{array}$ & $\begin{array}{c}\text { Empty } \\
\text { ETT Seen }\end{array}$ & Trach & Esoph & \\
\hline 9 & $\begin{array}{l}\text { Trach } \\
\text { Esoph }\end{array}$ & & $\begin{array}{l}1 \\
2\end{array}$ & $\begin{array}{c}\text { Empty } \\
\text { ETT Seen }\end{array}$ & Trach & Esoph & \\
\hline 10 & $\begin{array}{l}\text { Trach } \\
\text { Esoph }\end{array}$ & & $\begin{array}{l}1 \\
2\end{array}$ & $\begin{array}{c}\text { Empty } \\
\text { ETT Seen }\end{array}$ & Trach & Esoph & \\
\hline
\end{tabular}

Fig. 6 Learner Evaluation Form

standard-of-care methods. The clinical team will be accompanied by an independent research POCUS team, consisting of a POCUS user and a research assistant. The POCUS user will be a healthcare provider who has successfully completed phase 1 of the study.

The research POCUS team will be completely independent of the clinical team and will not provide them with results of the POCUS assessment. After completing the POCUS examination, the POCUS user will announce "complete" (to notify the research assistant for timing purposes) and will leave the room to record their findings. This will ensure that the clinical team is not affected by the result of our research test (which has unknown accuracy in this population). The POCUS user will have $30 \mathrm{~s}$ to complete the POCUS examination, after which the research assistant will notify them that their time is up. The research assistant will be present during the entire intubation and will record the time it takes for ETT confirmation via ultrasound and standard-of-care methods. If the patient needs more than one intubation attempt, then this information will be recorded by the research assistant, but the POCUS exam will only be performed with the first attempt.

\section{Data management and quality control}

Phase 1 data will be collected using the structured assessment of technical skills (see Fig. 5), the learner evaluation form (see Fig. 6), a learner demographic form (Fig. 7), and a learner feedback form (Fig. 8). These paper forms will then be transcribed into the Research Electronic Data Capture (REDCap) online tool. Phase 2 data will be collected by experienced female nurses who will be employed and trained for the study. Female nurse will be hired due to cultural acceptability, allowing access to the LR/OR. The data collector nurses will operate independently of both the clinical and POCUS teams, and will be available $24 \mathrm{~h}$ each day. They will initially collect intubation data using a patient data collection form (Fig. 9), then immediately transcribe the forms into REDCap.

For quality control, each POCUS examination will be recorded as a deidentified Mpeg4 video file on the POCUS tablet. These files will be sent via a secure 


\begin{tabular}{|c|c|}
\hline Name & \\
\hline Age & $\square \quad$ Prefer not to answer \\
\hline Gender & $\square \quad$ Prefer not to answer \\
\hline What is your professional designation? & $\begin{array}{ll} & \text { Staff Neonatologist } \\
\square & \text { Fellow in Neonatology } \\
\square & \text { General Pediatrician } \\
\square & \text { Paediatric Resident } \\
& \text { Other ( specify) }\end{array}$ \\
\hline $\begin{array}{l}\text { If you are a staff physician, how many years } \\
\text { have you been in practice (after completing } \\
\text { training) }\end{array}$ & Years in practice \\
\hline $\begin{array}{l}\text { Have you received or taken part in any } \\
\text { specific training on point of care ultrasound } \\
\text { (POCUS)? }\end{array}$ & $\begin{array}{ll}\square \quad \text { Yes } \\
\square \quad \text { No }\end{array}$ \\
\hline $\begin{array}{l}\text { If yes, have you been trained in airway } \\
\text { ultrasonography? }\end{array}$ & $\begin{array}{l}\square \text { Yes } \\
\square \quad \text { No }\end{array}$ \\
\hline $\begin{array}{l}\text { Prior to this training, were you aware the } \\
\text { point of care ultrasound could be used for } \\
\text { endotracheal tube placement confirmation? }\end{array}$ & $\begin{array}{ll}\square & \text { Yes } \\
\square & \text { No }\end{array}$ \\
\hline $\begin{array}{l}\text { On average, how many deliveries do you } \\
\text { attend per month? }\end{array}$ & $\begin{array}{ll}\square & <5 \\
\square & 5-10 \\
\square & 11-20 \\
\square & 20+ \\
\end{array}$ \\
\hline $\begin{array}{l}\text { On average, how many intubations do you } \\
\text { perform on newborns ( } 28 \text { days and younger) } \\
\text { each month? }\end{array}$ & $\begin{array}{ll}\square & <10 \\
\square & 11-30 \\
\square \quad 31-50 \\
\square & 50+ \\
\end{array}$ \\
\hline $\begin{array}{l}\text { On average, how many newborns ( } 28 \text { days } \\
\text { and younger) do you care for each month? }\end{array}$ & $\begin{array}{ll}\square & <10 \\
\square \quad 11-30 \\
\square \quad 31-50 \\
\square \quad 50+ \\
\end{array}$ \\
\hline
\end{tabular}

Fig. 7 Learner Demographic Form

online file transfer program to a non-AKUH paediatric intubation POCUS expert who was not part of study interventions and is blinded to any patient details. The expert will rate each video for high or low quality images, and will record an interpretation of esophageal or tracheal intubation.

\section{Measurement of outcomes}

The primary outcome is diagnostic accuracy [(true positives+ true negatives)/total N] of POCUS (performed by healthcare providers with a brief education in intubation POCUS) for identifying tracheal versus esophageal intubation in newborns. The reference standard will be a composite of standard-of-care methods (auscultation, colorimetric capnography, and chest $\mathrm{x}$-ray). The secondary outcome is the time difference between POCUS and standard-of-care methods in determining ETT location. The tertiary outcomes include the level of agreement between POCUS users and standard-of-care methods, the level of agreement between POCUS users and a
POCUS expert, and the level of agreement between the POCUS expert and standard-of-care methods.

\section{Hypotheses}

Neonatal health care providers trained on a novel ultrasound simulator in ETT localization will be $>95 \%$ accurate, compared to an external POCUS expert, when performing POCUS on intubated neonates, and will produce a result in less time than the current standard-ofcare methods for ETT localization.

\section{Sample size}

Phase 1 will involve 60 healthcare providers who attend deliveries and are exclusively involved in newborn care, which include 6 neonatal attending physicians, 8 neonatal fellows, 38 postgraduate medical trainees, and 8 senior nursing staff. These providers are all skilled in standard-of-care methods for ETT confirmation. Each 2-hour training session involves 15 participants, and the sessions are conducted 4 times. 


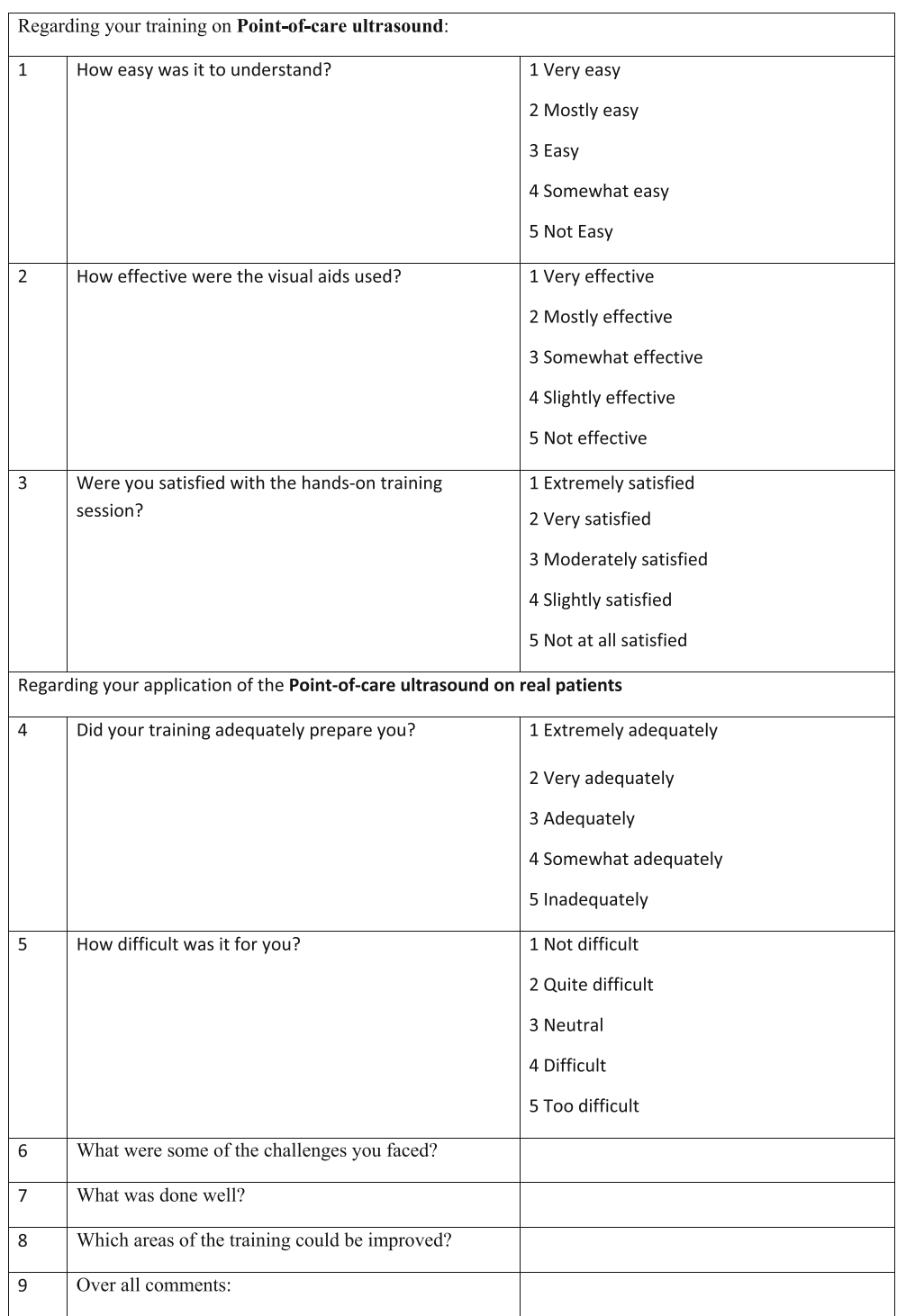

Fig. 8 Learner feedback form

The phase 2 sample size calculation indicates that a total of 292 neonatal intubations are required. There are approximately 5000 deliveries per year at $\mathrm{AKUH}$, of which approximately $12 \%$ (600) of these newborns are admitted to the NICU. Approximately 70\% (420) of these patients require intubation in a given year. The rates of incorrect esophageal intubation reported in the most recent multicenter study ranged from 1 to $12 \%$ [13]. Using a conservative rate of $5 \%$, the sample size was calculated for the primary outcome (accuracy) using a two-sided equivalence test for correlated proportions. The standard proportion of correct tracheal intubation was assumed to be $95 \%$ and the maximum allowable difference between POCUS and standard-of-care methods was set to $0.05(\alpha)$. To achieve
$80 \%$ power at a $5 \%$ significance level, a total of 222 participants are needed for the primary outcome.

For the secondary outcome (time difference between POCUS and standard-of-care methods), using a conservative time difference of $5 \mathrm{~s}$, we estimate a total sample size of 252 newborns will be needed to achieve a twosided alpha of 0.05 and power of $80 \%$.

Sample size estimates for the tertiary outcome are based on the kappa ( $\mathrm{\kappa}$ ) statistic for measuring agreement between POCUS and standard-of-care methods. Assuming 95\% true agreement and a kappa statistic of 0.90, a sample size of 292 subjects results in a two-sided 95\% confidence interval with a width of 0.100 (i.e. $\mathrm{k}=0.85-0.95$ ) using the Cohen's large-sample formula for standard deviation of $\kappa$. 

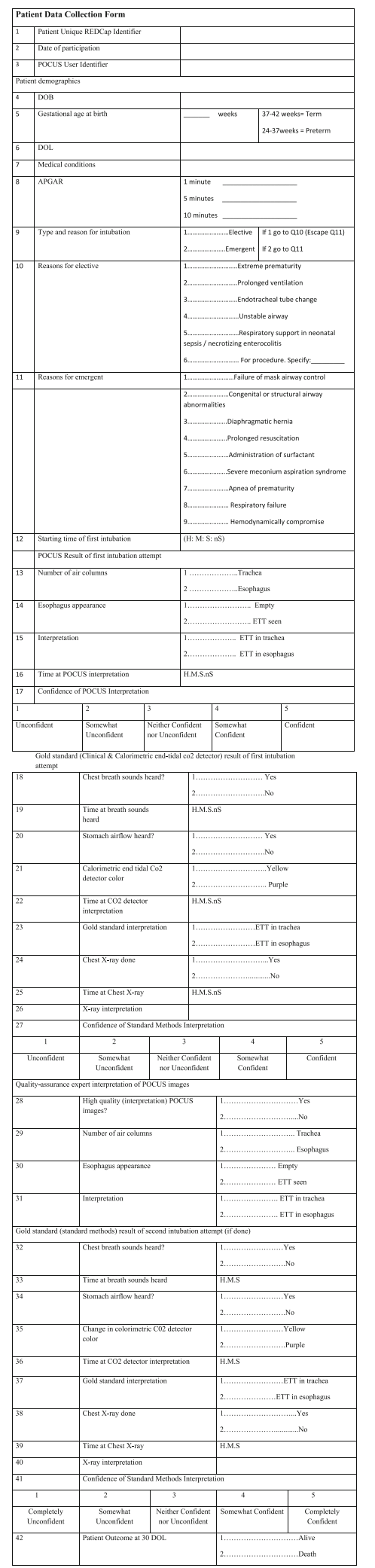

Fig. 9 Patient Data Collection Form
Therefore, to achieve all study objectives, a minimum sample size of $n=292$ will be targeted.

\section{Timing}

We estimate that both study phases can be completed in approximately 12 months.

\section{Data processing and analysis}

Key study variables will be summarized univariately using means/standard deviations/histograms and frequencies/ proportions as appropriate.

We will calculate sensitivity, specificity, positive and negative predictive values, positive and negative likelihood ratios, and the $\mathrm{K}$ statistic (all with 95\% confidence intervals) using standard formulas for a binomial proportion.

The mean time difference between POCUS and standard-of-care methods will be analyzed using paired sample t-tests. If the time distribution is skewed, we will examine median time differences using Wilcoxon signedrank tests for matched data.

Data analysis will use SPSS statistical software, with the type 1 error rate set to 0.05 . A subgroup analysis of premature newborns status will be performed as sample size permits. We will also analyze user accuracy as a function of number of POCUS exams performed during the study. An ad-hoc power analysis will be performed on stratified effects at study completion.

\section{Discussion}

Intubation during newborn resuscitation is a critical lifesaving skill that is difficult to learn and perform. Unsuccessful intubation can lead to devastating consequences, and one of the most common reasons for intubation failure of is esophageal placement of an ETT [26]. Ultrasound provides a simple, safe and effective method of differentiating between esophageal and tracheal intubations, but has not yet achieved widespread use in newborn resuscitation [27].

To our knowledge, only two previous studies have utilized ultrasound in real time for the confirmation of ETT position with comparison to standard-of-care methods $[28,29]$. The first study included 16 NICU intubations and found a sensitivity of $92 \%$ and a specificity of $100 \%$ for ultrasound compared to capnography. Ultrasound was significantly slower than capnography, but significantly faster than $\mathrm{x}$-ray [28]. The second study included 10 NICU intubations and $100 \%$ sensitivity and specificity for ultrasound compared to capnography, with ultrasound results generated significantly faster than x-ray [29]. Our study represents the largest study to date examining the use of POCUS for newborn intubations.

The second major strength of this study is the development and validation of a novel ultrasound simulator. Using materials costing only $\$ 2$, an additional potential benefit of this study is to demonstrate that this simulator 
can be used to train healthcare providers in this important ultrasound skill. Simulators have the advantage of allowing providers to learn and practice skills prior to their application on patients, and other ultrasound simulators have been designed for this purpose [30]. Additional strengths of this study include 1) recorded images with quality assurance 2) different types of healthcare workers involved in being trained 3) extended follow-up period to measure skills retention, and 4) empowering health care providers to read and interpret images in real time thereby saving critical time and need for specialized human resources (radiologists).

The major limitation of this study is related to ultrasound cost as it pertains to the generalizability of the study. Although the simulator is low-cost, the ultrasound equipment is expensive and will not be able to be purchased in many low and middle-income settings at current price points. Over time, however, this will change as ultrasound technology continues to be more accessible. It is also important to note that ultrasound has a wide variety of applications in the NICU [31], and will increasingly become available for use.

\section{Abbreviations}

AKUH: Aga Khan University Hospital; CO2: Carbon dioxide; ETT: Endotracheal tube; LR/OR: Labour room/operating room; NICU: Neonatal intensive care unit; POCUS: Point of care ultrasound; SD: Standard deviation

\section{Acknowledgments}

The authors would like to thank the NICU staff, especially Simon Demas and Mehjabeen Aziz, for their assistance in organizing the training sessions at AKUH, and for data entry. The authors would also like to thank Dr. Nadia Akseer for her assistance in sample size calculations.

\section{Authors' contributions}

HSM and MOT conceived of the study and drafted the manuscript. KQA, SKM, SBS and SA participated in the design of the study and revised the manuscript critically for intellectual content. All authors have read and approved the final manuscript.

\section{Funding}

This study is funded by Grand Challenges Canada Stars in Global Health project number: ST-POC- 1707-06658.

Contact information: Grand Challenges Canada, MaRS Centre, West Tower, 661 University Avenue, Suite 1720, Toronto, ON M5G 1 M1. Telephone +1416-583-5821.

E-mail:info@grandchallenges.ca.

Grand Challenges Canada provided the peer review for this protocol. The funder had no role in study design; collection, management, analysis, and interpretation of data; writing of the report; and the decision to submit the report for publication.

\section{Availability of data and materials}

Given the sensitive nature of the data which includes ultrasound video clips of newborns, the data is only available to two co-authors (MT and KQA) via secured file sharing platform housed at The Hospital for Sick Children. The authors plan to publish aggregate study results in peer-reviewed journals and present the findings at conferences.

\section{Ethics approval and consent to participate}

This study received approval from the Research Ethics Board at Sickkids Hospital (REB\#: 1000057021) and the AKUH Ethical Review Committee (ERC\#:4927-Ped-ERC-17) and the National Bioethics Committee of Pakistan (4-87/NBC-319/18/552). Any amendments to the study protocol will be submitted to both hospital committees for approval.
In phase I of this study, all healthcare providers will provide written informed consent prior to participating in the study. For phase II of the study two separate protocols have been established for the use of ultrasound during intubations. Given the time-critical nature of emergency intubation, we will not be able to complete informed consent for research participation with families of these newborns. Therefore, we will utilize a deferred consent procedure and have ensured our study satisfies TCPS-2 Article 3.7 A for deferred consent. We will obtain written informed consent from parents after intubation and stabilization of the newborn. While not as time-critical as emergency intubations, semi-elective intubation can proceed rapidly and be associated with stressful timing for parents. These circumstances may interfere with the ability to provide informed consent at the time of a semi-elective intubations, so families will be approached for written informed consent at the time of admission to the NICU. Given that the data collected in the POCUS intervention does not change standard of care practices, a data monitoring committee is not needed for this study.

\section{Consent for publication}

Not applicable.

\section{Competing interests}

The authors declare that they have no competing interests.

\section{Author details}

'Division of Pediatric Emergency Medicine, McMaster Children's Hospital, McMaster University, 1280 Main Street West, HSC-2R104, Hamilton, ON L8S 4K1, Canada. ${ }^{2}$ Division of Pediatric Emergency Medicine, Emergency Point-of-Care Ultrasound Program, Hospital for Sick Children, 555 University Avenue, Toronto, ON M5G 1X8, Canada. ${ }^{3}$ Department of Paediatrics \& Child Health, Aga Khan University, Stadium Road, Karachi 74800, Pakistan. ${ }^{4}$ Division of Infectious Diseases and Centre for Global Child Health, Hospital for Sick Children, Department of Pediatrics Faculty of Medicine, 555 University Avenue, Toronto, ON M5G1X8, Canada.

Received: 25 June 2019 Accepted: 12 September 2019

Published online: 13 November 2019

\section{References}

1. Jehan I, Harris H, Salat S, Zeb A, Mobeen N, Pasha O, et al. Neonatal mortality, risk factors and causes: a prospective population-based cohort study in urban Pakistan. Bull World Health Organ. 2009;87(2):130-8.

2. Pakistan statistics summary (2002 - present). WHO. 2018. Available from: http://apps.who.int/gho/data/node.country.country-PAK. Accessed 2 Dec 2018.

3. Lawn JE, Cousens S, Zupan J. Lancet neonatal survival steering T. 4 million neonatal deaths: when? where? why? Lancet. 2005;365(9462):891-900.

4. Halloran DR, McClure E, Chakraborty H, Chomba E, Wright LL, Carlo WA. Birth asphyxia survivors in a developing country. J Perinatol. 2009;29(3):243-9.

5. Askin DF. Complications in the transition from fetal to neonatal life. J Obstet Gynecol Neonatal Nurs. 2002;31(3):318-27.

6. Hillman NH, Kallapur SG, Jobe AH. Physiology of transition from intrauterine to extrauterine life. Clin Perinatol. 2012;39(4):769-83.

7. Stenson BJ, Boyle DW, Szyld EG. Initial ventilation strategies during newborn resuscitation. Clin Perinatol. 2006;33(1):65-82 vi-vii.

8. American Academy of Pediatrics/American Heart A. American Academy of Pediatrics/American Heart Association clarification of statement on cardiovascular evaluation and monitoring of children and adolescents with heart disease receiving medications for ADHD: May 16, 2008. J Dev Behav Pediatr. 2008;29(4):335.

9. Schmolzer GM, Kumar M, Pichler G, Aziz K, O'Reilly M, Cheung PY. Noninvasive versus invasive respiratory support in preterm infants at birth: systematic review and meta-analysis. BMJ. 2013;347:f5980.

10. Carbajal R, Eble B, Anand KJ. Premedication for tracheal intubation in neonates: confusion or controversy? Semin Perinatol. 2007;31(5):309-17.

11. Schmolzer GM, O'Reilly M, Davis PG, Cheung PY, Roehr CC. Confirmation of correct tracheal tube placement in newborn infants. Resuscitation. 2013;84(6):731-7.

12. Peterson J, Johnson N, Deakins K, Wilson-Costello D, Jelovsek JE, Chatburn R. Accuracy of the 7-8-9 rule for endotracheal tube placement in the neonate. J Perinatol. 2006;26(6):333-6. 
13. Foglia EE, Ades A, Sawyer T, Glass KM, Singh N, Jung P, et al. Neonatal intubation practice and outcomes: an international registry study. Pediatrics. 2019;143(1). https://doi.org/10.1542/peds.2018-0902.

14. Sitzwohl C, Langheinrich A, Schober A, Krafft P, Sessler DI, Herkner $\mathrm{H}$, et al. Endobronchial intubation detected by insertion depth of endotracheal tube, bilateral auscultation, or observation of chest movements: randomised trial. BMJ. 2010;341:c5943.

15. Roberts WA, Maniscalco WM, Cohen AR, Litman RS, Chhibber A. The use of capnography for recognition of esophageal intubation in the neonatal intensive care unit. Pediatr Pulmonol. 1995;19(5):262-8.

16. Schmolzer GM, Poulton DA, Dawson JA, Kamlin CO, Morley CJ, Davis PG. Assessment of flow waves and colorimetric $\mathrm{CO} 2$ detector for endotracheal tube placement during neonatal resuscitation. Resuscitation. 2011;82(3):307-12.

17. Aziz HF, Martin JB, Moore JJ. The pediatric disposable end-tidal carbon dioxide detector role in endotracheal intubation in newborns. J Perinatol. 1999;19(2):110-3.

18. Blank D, Rich W, Leone T, Garey D, Finer N. Pedi-cap color change precedes a significant increase in heart rate during neonatal resuscitation. Resuscitation. 2014;85(11):1568-72.

19. Das SK, Choupoo NS, Haldar R, Lahkar A. Transtracheal ultrasound for verification of endotracheal tube placement: a systematic review and metaanalysis. Can J Anaesth. 2015:62(4):413-23.

20. Chou EH, Dickman E, Tsou PY, Tessaro M, Tsai YM, Ma MH, et al. Ultrasonography for confirmation of endotracheal tube placement: a systematic review and meta-analysis. Resuscitation. 2015;90:97-103.

21. Galicinao J, Bush AJ, Godambe SA. Use of bedside ultrasonography for endotracheal tube placement in pediatric patients: a feasibility study. Pediatrics. 2007;120(6):1297-303.

22. Chou HC, Chong KM, Sim SS, Ma MH, Liu SH, Chen NC, et al. Real-time tracheal ultrasonography for confirmation of endotracheal tube placement during cardiopulmonary resuscitation. Resuscitation. 2013;84(12):1708-12.

23. Chou HC, Tseng WP, Wang CH, Ma MH, Wang HP, Huang PC, et al. Tracheal rapid ultrasound exam (T.R.U.E.) for confirming endotracheal tube placement during emergency intubation. Resuscitation. 2011;82(10):1279-84.

24. Schmolzer GM, Roehr CC. Techniques to ascertain correct endotracheal tube placement in neonates. Cochrane Database Syst Rev. 2014;(9): CD010221. https://doi.org/10.1002/14651858.CD010221.pub2.

25. Seguin J, Tessaro MO. A simple, inexpensive phantom model for intubation ultrasonography training. Chest. 2017;151(5):1194-6.

26. O'Shea JE, Loganathan P, Thio M, Kamlin COF, Davis PG. Analysis of unsuccessful intubations in neonates using videolaryngoscopy recordings. Arch Dis Child Fetal Neonatal Ed. 2018;103(5):F408-F12.

27. Sharma D, Tabatabaii SA, Farahbakhsh N. Role of ultrasound in confirmation of endotracheal tube in neonates: a review. J Matern Fetal Neonatal Med. 2019;32(8):1359-67.

28. Alonso Quintela P, Oulego Erroz I, Mora Matilla M, Rodriquez Blanco S, Mata Zubillaga D, Regueras Santos L. Usefulness of bedside ultrasound compared to capnography and X-ray for tracheal intubation. An Pediatr (Barc). 2014;81(5):283-8.

29. Mora-Matilla M, Alonso-Quintela P, Oulego-Erroz I, Rodriguez-Blanco S, Gautreaux-Minaya S, Mata-Zubillaga D. Is ultrasound a feasible tool to verify endotracheal tube position in neonates? Resuscitation. 2013;84(1):e19-20.

30. Ng V, Plitt J, Biffar D. Development of a novel ultrasound-guided Peritonsillar abscess model for simulation training. West J Emerg Med. 2018; 19(1):172-6.

31. Chen SW, Fu W, Liu J, Wang Y. Routine application of lung ultrasonography in the neonatal intensive care unit. Medicine (Baltimore). 2017;96(2):e5826.

\section{Publisher's Note}

Springer Nature remains neutral with regard to jurisdictional claims in published maps and institutional affiliations.

Ready to submit your research? Choose BMC and benefit from:

- fast, convenient online submission

- thorough peer review by experienced researchers in your field

- rapid publication on acceptance

- support for research data, including large and complex data types

- gold Open Access which fosters wider collaboration and increased citations

- maximum visibility for your research: over $100 \mathrm{M}$ website views per year

At $\mathrm{BMC}$, research is always in progress.

Learn more biomedcentral.com/submissions 